\title{
The Importance of Maintaining the Momentum of Telemedicine after COVID-19
}

\author{
Negar Salehi ${ }^{1}$, Saima Karim², Aisha Siraj ${ }^{2 *}$ \\ ${ }^{1}$ Department of Cardiology, University of Arkansas for Medical Sciences, 4301 W Markham S, Little Rock, Arkansas \\ ${ }^{2}$ Heart and Vascular Institute, Case Western Reserve University/MetroHealth Medical Center Campus, 2500 Metrohealth Medical Center, Cleveland, \\ Ohio
}

*Corresponding Author: Aisha Siraj, Heart and Vascular Institute MetroHealth Medical Center, Case Western Reserve University 2500 MetroHealth Drive Cleveland.

Received Date: November 11, 2020; Accepted Date: January 11, 2021; Published Date: January 27,2021

Citation: Negar Salehi, Saima Karim, Aisha Siraj., (2021) The Importance of Maintaining the Momentum of Telemedicine after COVID-19. J. Clinical Cardiology and Cardiovascular Interventions, 4(2); Doi:10.31579/2641-0419/107

Copyright: (c) 2021 Aisha Siraj, This is an open-access article distributed under the terms of the Creative Commons Attribution License, which permits unrestricted use, distribution, and reproduction in any medium, provided the original author and source are credited.

\begin{abstract}
:
Telemedicine has been in existence for years with little traction for global mainstream medicine. COVID19 has exposed the importance of providing continued care for patients while minimizing the risk of exposure during pandemics. There has been robust growth in the use of telemedicine since the pandemic began rendering care possible while providing safety to patients and healthcare workers. Various digital applications are available to facilitate telehealth. Even in the absence of a pandemic, telemedicine allows the care of patients who may live in remote areas, or those who have issues with transportation or comorbidities prohibiting ambulation.

This study is based on a questionnaire of providers who have been exposed to telemedicine during COVID19. A survey was shared in social media forums involving groups of physicians, and nurse practitioners who were willing to take the survey.

Telemedicine is one of the best approaches to handling situations like pandemics or disease surges. In these circumstances, a virtual visit is beneficial for social distancing if a laptop, smartphone, or tablet is available, along with an internet or cellular coverage.

Based on this survey conducted for healthcare workers from a variety of specialties, there was a considerable impetus for the continued benefit from telemedicine as an alternative to in-person visits for selected patients. Ongoing improvements in quality of applications/tools, education, and the cost are essential to maintain the benefits of telemedicine. There is also an ongoing necessity for vast improvements in healthcare policies and reimbursements.

Key Words: telemedicine; telephone encounter; video visit; virtual visit

Running Head: Importance of Telemedicine after COVID-19
\end{abstract}

\section{Background}

Despite the existence of telemedicine for years, COVID 19 has accelerated and highlighted the importance of providing continued care for patients while minimizing the risk of exposure during a pandemic. The lack of widespread adoption of telemedicine before COVID-19 is partly because of lack of regulatory framework regarding stepwise initiation, integration into guidelines for practice (especially in conjunction with electronic medical records), funding framework for reimbursement of services rendered, standardized triaging of care provided, education of public en masse, and safe data sharing mechanism within facilities. While COVID-19 has caused an acceleration in the use of telemedicine tools, there have been pandemics globally prior to COVID-19, and there is a likely possibility of other pandemics after COVID-19. [1,2]

There has been robust growth in the use of telemedicine since the pandemic began, and rendering care has become possible while providing safety to patients, patient's relatives, providers, and other healthcare workers. It has been possible to continue limited in-person appointments due to decreased volume with continued social distancing in patient care areas and waiting rooms. Additionally, telemedicine has reduced the need for personal protective equipment for providers and patients, given the national shortage. The tools for telemedicine include telephone, videovisits, smartphone data-apps, modules linked with an electronic medical record (EMR), and other methods to a smaller degree. Examples of applications that integrate into EMR include video visits on Epic, MDlink, American Well, Polycom RealPresence, CyraCom. Examples of applications that do not integrate into EMR are SnapMD, Doximity, Zoom, FaceTime, Skype, and WhatsApp. The health insurance portability and accountability action(HIPAA) compliant applications among these are Epic, Doximity, MDLink, Polycom RealPresence, etc.

Even in the absence of a pandemic, telemedicine allows the care of patients who are either unable to get transportation, have comorbidities that prohibit ambulation, or those who live in remote areas without access to providers. Unfortunately, educational, and socioeconomic gaps may become more prominent between those who can afford or understand how to use smartphones, tablets, computers with video access versus those who cannot learn how to use or afford these modalities. The learning curve of new technology with regards to telehealth may also be prohibitive based on prior exposure and age as well. However, even a 
telephone encounter may provide ongoing care for these individuals despite the lack of possibility of some physical exam features that can be detected on video visits. Patient barriers to telemedicine also include the desire to see the provider during periods of illness, inability to communicate with an established provider (versus a new provider without an established relationship), and lack of awareness or access to telemedicine. As far as providers are concerned, the barriers to care include similar problems of patient education, patient preference for an in-person visit, issues with appropriate triaging, access to technology, and appropriately approved data-apps. Challenges with network, reimbursement, decreased productivity, inability to provide physical exam except for certain elements that can be visualized via video visits are extra barriers. [2]

Triaging and workflow should be tailored to the field of medicine for telehealth to be effective. In places, such as urgent care and emergency rooms, "forward triage" allows for effective prospective sorting of urgency before arrival for surge control via screening tools. It also allows for patients with high-risk features of COVID-19 to be isolated immediately upon arrival. [3]

On the other hand, patients who are mildly ill get their care via telephone, video encounters via various smartphones or apps. Triage is imperative regarding the need for in-person visits versus virtual visits that occur in primary care and specialty offices with the use of protocols that are set in place in most practices depending on diagnosis, the severity of symptoms, urgency of care needed, and number or severity of comorbidities. [4]

There are two fronts to telemedicine that are acknowledged: asynchronous (with no concurrent communication between provider and patient); and synchronous (live communication between provider and patient). Asynchronous care can include remote monitoring of devices or chronic conditions, exchange of messages via EMR, results of tests being reported/mailed, medication refills, and management. Synchronous care includes in-person, virtual, or telephone visits with a provider. It is necessary to distinguish between these as the asynchronous telemedicine has been in place and utilized widely by most practices before the pandemic. [5]

Current requirements for telehealth visits include documentation of history, chief complaint, review of systems, past medical history, assessment and plan, level of complexity, mention of any additional workup, total time spent on the encounter (with at least $50 \%$ of the time spent in counseling and coordinating care), and examination items (on video visits). Vital signs such a heart rate, blood pressure, respiratory rate, etc. can be added if taken by the patient at home. Adaption of telemedicine within an orthopedic department leads to acceleration to $50 \%$ of the average clinical volume within 2 weeks with an indication for in-person visit triage list. Checklists to ensure the appropriate use of electronic medical regards, cameras/microphones, provision of privacy, templates, patient and provider education, appropriate triaging, and adherence to regulations should be made to allow for the feasibility of rapid implementation. [6]

Some factors that are involved in the evolution of telemedicine to make it sustainable, safe and widespread include adequate training of patients/providers/ancillary staff/schedulers/nurses, protocolization triaging, appropriate documentation guides and smart phrases, data protection, confidentiality, and safe data sharing practices. Additionally, reimbursement may be a critical factor in the continuity of the provision of telemedicine as well.[7]

Even though telehealth has revolutionized medicine, the provider's perspective on its need, user-friendliness, limitations, desire for continuity is missing. We surveyed to involve providers in the discussion to see what their perspective of appropriateness, necessity, and suitability of telehealth in their field is.

\section{Result}

This study was based on a questionnaire of providers who have been exposed to the concept of telemedicine during COVID-19. A survey was shared in social media forums involving unbiased groups of physicians, nurse practitioners (NPs), and advanced practice nurse practitioners (APRNs) who were willing to take the survey. In the end, 200 providers from different fields of medicine took the survey about telemedicine.

Among those that took the survey, $91.9 \%$ were female, $86.5 \%$ had an MD degree, and $0.5 \%$ physician had DO degree. There were also $1 \%$ APRNs and $1 \%$ NPs. $86.5 \%$ had completed their training and were practicing independently, while $13.5 \%$ were still in training. A myriad of medical specialties was represented in the survey, which are highlighted in (figure 1).

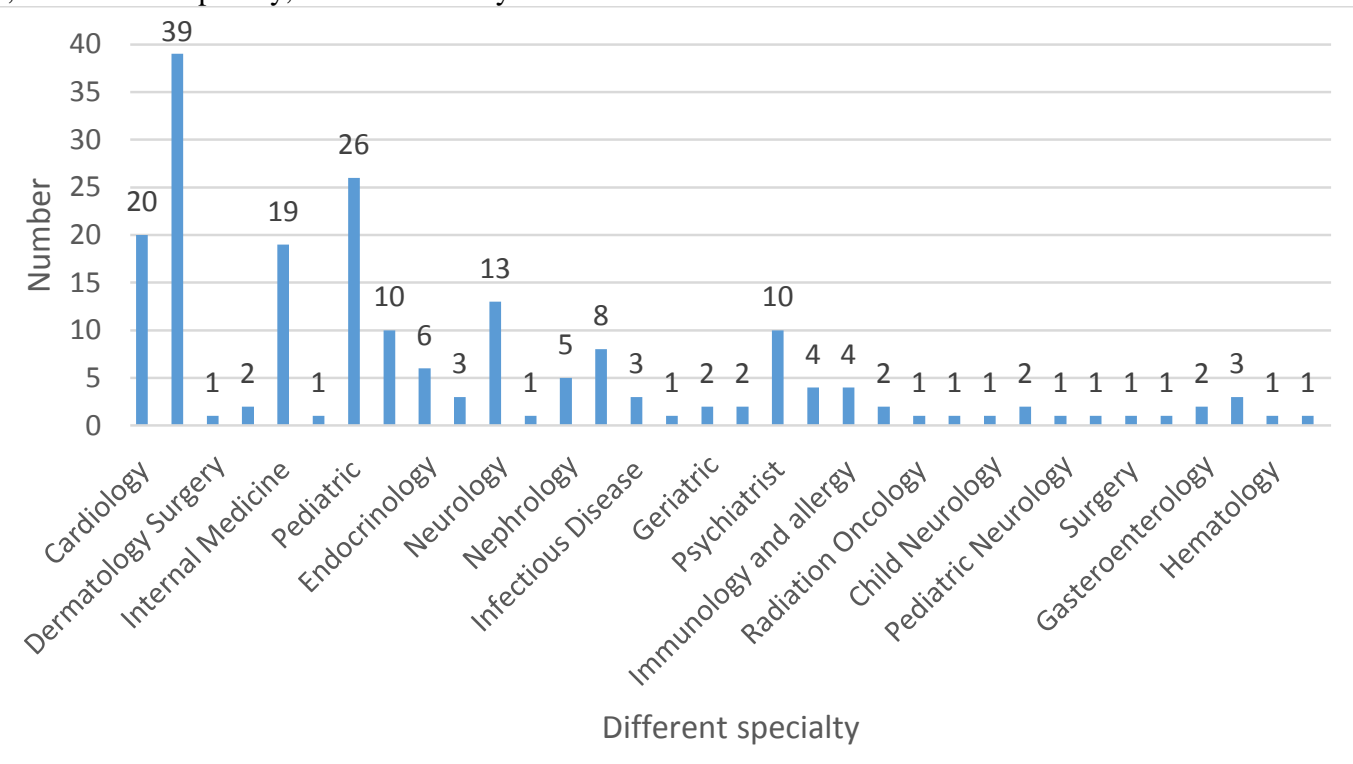

Figure-1: Different specialties in the study 
Primary care (including Family Medicine, Internal Medicine, Pediatrics) accounted for $42 \%$ of those surveyed. Other larger subsets of specialties represented included Cardiology (10\%), Neurology (6.5\%), and Psychiatry (5\%).

$96.5 \%$ of those who took the survey were performing telemedicine. Based on this survey, Doximity and Epic videos are the most common modalities used for telemedicine ( $18.75 \%$ and $18.23 \%$, respectively). However, Zoom video (17.19\%), telephone encounter (13.02\%) wre also common along with Facetime, Google due, and Doximity audio, which were less than 5\% each. (Figure-2) $71.79 \%$ of the medical staff agreed that follow up patients were the most suitable type of patients for telemedicine.
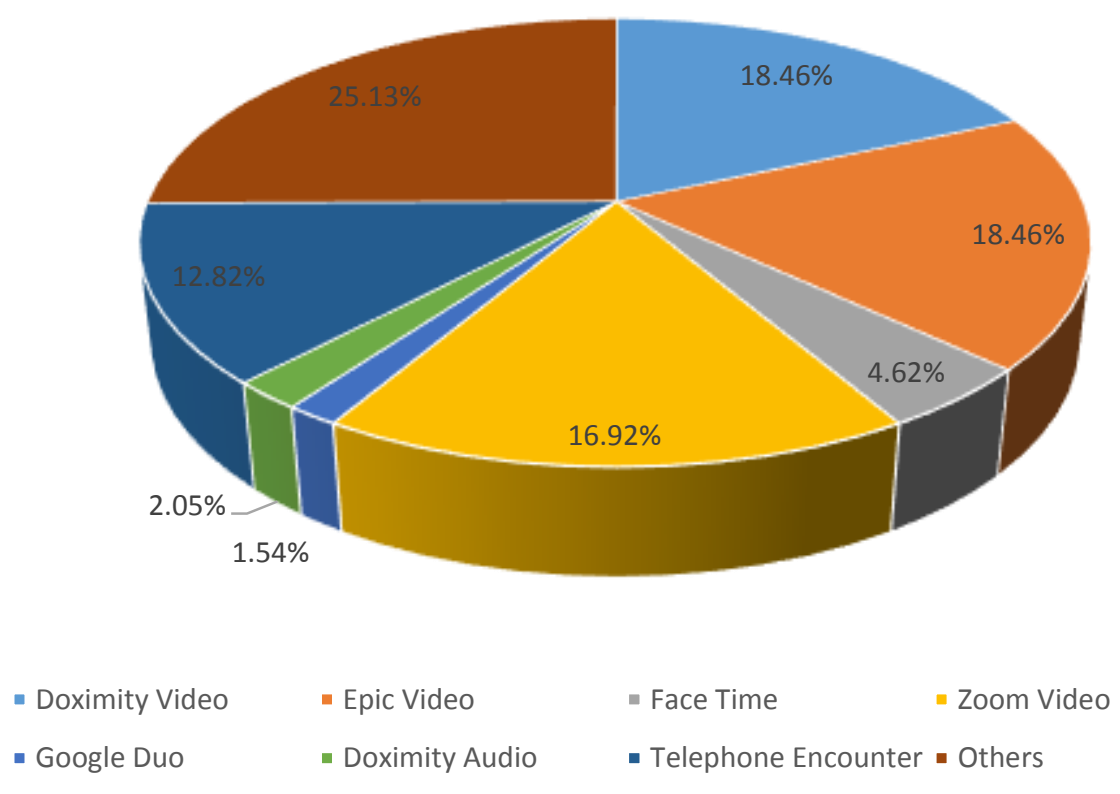

Figure-2: Different applications has been used for telemedicine base on this survey

The second most appropriate category of patients for telemedicine were those with less than 3 comorbidities (51.79\%). However, $25 \%$ of those surveyed believed that there is no difference between a virtual/telephone/app visit or regular clinic visit. Among those of took the survey, $47.7 \%$ thought that doing a physical exam in their specialty is not necessary, while $42.2 \%$ emphasized on the importance of physical exams on their visits. The overwhelming majority of health care workers $(96.5 \%)$ thought that telemedicine was a good mode for patient encounter, and most of them agreed that this needs to be part of the training curriculum (90\%).

There was an agreement that ancillary staff such as schedulers, triage nurses, clinic nurses, and medical assistants, etc. would continue to provide an invaluable service with telemedicine (81.63\%). 93\% agreed that telemedicine should be a viable option after the pandemic as well.

Telemedicine seemed to have variable effects on productivity of providers, $41 \%$ of the providers agreed that telemedicine increased their work productivity, and $41 \%$ mentioned that their efficiency remains the same. At the same time, about $50 \%$ of responders agreed that reimbursement was either good or reasonable, while $26.15 \%$ thought that is could be better, and $15.9 \%$ believed that it needed significant improvement. (Figure-3) 71\% thought that pandemic had not affected their research productivity, while $21.5 \%$ agreed that this pandemic decreased their productivity. Interestingly, $60 \%$ of survey takers reported that telemedicine helped to have more balance between work and their life. 


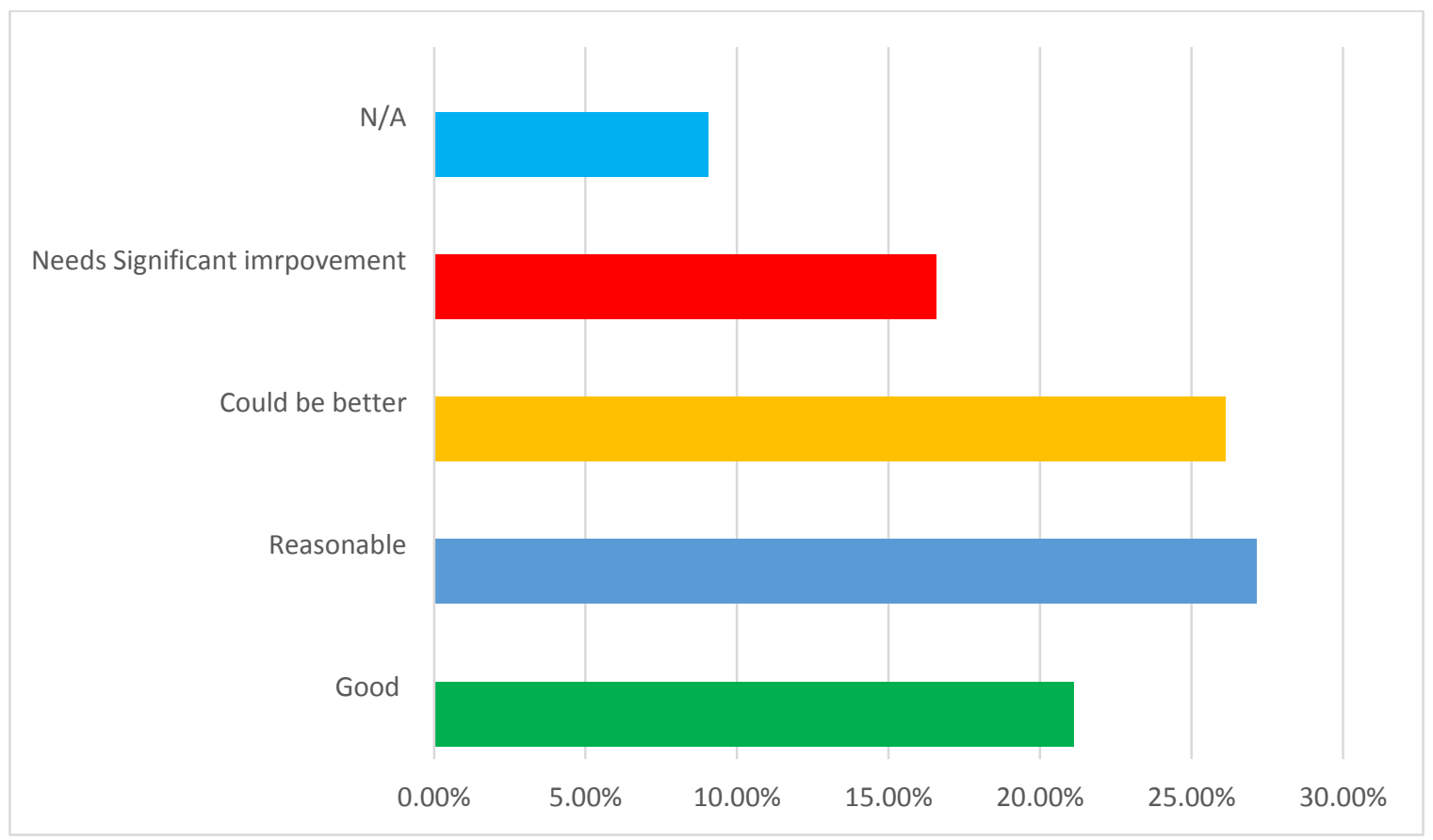

Figure-3: Reimbursement of Telemedicine

\section{Discussion:}

Telemedicine is a concept that started in the mid-19 $19^{\text {th }}$ century. Communication such as the telegraph and the telephone opened the possibility of remote medical care. In 1924, when the radio was not widely used and television was not yet invented, an avant garde illustration of a family communicating with their physician via a video screen was presented in Radio News Magazine. [8]

The novel version of telemedicine was created in the mid-20 $20^{\text {th }}$ century from aerospace and military communication technology. [9] At that time, telemedicine defined by electrocardiogram monitoring, the transmission of radiology images, supervision of students by advanced practice professionals, and remote education. Data transfer was expensive, and appropriate equipment was lacking leading to hindrance in advancement until the late 1980-1990s when these barriers were simplified. [9]

So far, telemedicine has been successfully used in several disasters liked responses to the Texas hurricane and the Gulf Coast disasters in the fall of 2017. These trials confirmed the availability of access to physicians, but some challenges needed to be overcome. Most of the services, including the prescription of routine medications, evaluation of insect bites, wound infections, and mental health were managed successfully. [10]

Telemedicine is one of the best approaches to handling situations like pandemics or disease surges. In these circumstances, a virtual visit is beneficial for social distancing if a laptop, smartphone, or tablet is available, along with an internet or cellular coverage. Telemedicine utility during COVID-19 is the most recent urgency that has unveiled that virtual visits could be a reasonable replacement for at least $70-80 \%$ of follow up and some of the initial visits.

In this questionnaire, we asked healthcare workers from various specialties with different levels of education about telemedicine. The majority agreed that this could be an excellent tool that should continue after the pandemic with a need to include telemedicine in medical school, graduate, and post-graduate education to instruct regarding different aspects of patient selection, obtainment of appropriate information, the possibility of a physical exam, variety of remote testing/parameters that are feasible, need for escalation of care etc. One of the limitations of this survey was the absence of data and information from the patients' point of view. Another limitation was that this was a survey that was taken mainly by female healthcare providers. However, the questions asked were not gender-specific, and results were likely not affected by the low proportion of male participants, except for questions regarding work-life balance which innately affects the genders differently in the current healthcare structure.

The survey was instrumental in understanding that healthcare providers appreciate telemedicine and want it as a continued tool. Telemedicine can decrease the cost of healthcare, increase the level of patients' satisfaction, provide a better life-work balance for healthcare providers, increase access to care, and provide safe and efficient telephone or virtual visits during pandemics or other dire times. The risks of telemedicine include potential patient data safety, such as HIPPA compliance with tools used for telemedicine, creation of appropriate algorithms for proper patient selection, understanding of remote tools to supplement care (such as vitals done remote of smart devices), and knowledge of appropriateness of escalation of care if needed. The importance of advantages and disadvantages of telemedicine and traditional care should be understood by healthcare workers and patients to allow for safe delivery of care.

While everybody is mindful of the requirements for conventional visits, awareness of the tools and setting for telemedicine should also be widespread. HIPPA compliant devices and space that healthcare providers can use for telemedicine are necessary to have an uninterrupted private visit with patients with the provision of ancillary support. Additionally, access and knowledge of tools such as laptops, smartphones, or tablets, HIPPA compliant web-based devices, or applications are necessary for the patient as well.

Based on this survey conducted for healthcare workers from a variety of specialties, there was a considerable impetus for the continued benefit from telemedicine as an alternative to in-person visits for selected patients. 
Ongoing improvements in quality of telemedicine forums, supplemental tools, education, and the cost are essential to maintain the benefits of telemedicine. There is a necessity for vast improvements in healthcare policies and reimbursements that will allow for the use of telemedicine to benefit healthcare providers and patients in the future.

This article highlights the healthcare provider's perspective on the benefits of telemedicine, along with some of the challenges. COVID-19 has provided us with the opportunity to expand access and modes of care that we can provide our patients. We hope that the desires of healthcare workers during COVID19 serve as catalyst to further education and improvement in healthcare policies and reimbursement that will help telemedicine endure and expand beyond the pandemic.

\section{Highlights:}

- Telemedicine is a growing modality to for provision of care to patients during a pandemic with need for more education, better tools and reimbursement

- This is a healthcare providers point of view of telemedicine during COVID19

\section{Conflict of Interest Disclosures: None}

\section{References:}

1. Portnoy J, Waller M, Elliott T. Telemedicine in the Era of COVID-19. J Allergy Clin Immunol Pract. 2020 May;8(5):1489-1491.

2. Ohannessian R, Duong TA, Odone A. Global Telemedicine Implementation and Integration Within Health Systems to Fight the COVID-19 Pandemic: A
Call to Action. JMIR Public Health Surveill [Internet]. 2020 Apr 2 [cited 2020 Aug 8];6(2).

3. Hollander JE, Carr BG. Virtually Perfect? Telemedicine for Covid-19. N Engl J Med [Internet]. 2020 Mar 11 [cited 2020 Aug 8];

4. Hare N, Bansal P, Bajowala SS, Abramson SL, Chervinskiy S, Corriel R, et al. COVID-19: Unmasking Telemedicine. J Allergy Clin Immunol Pract [Internet]. 2020 Jun 27 [cited 2020 Aug 8];

5. Portnoy JM, Wu AC. Is Telemedicine as Effective as Usual Care? J Allergy Clin Immunol Pract. 2019 Jan $1 ; 7(1): 217-218$

6. Loeb AE, Rao SS, Ficke JR, Morris CD, Riley LH, Levin AS. Departmental Experience and Lessons Learned With Accelerated Introduction of Telemedicine During the COVID-19 Crisis. J Am Acad Orthop Surg [Internet]. 2020 Apr 14 [cited 2020 Aug 8];

7. Iyengar K, Jain VK, Vaishya R. Pitfalls in telemedicine consultations in the era of COVID 19 and how to avoid them. Diabetes Metab Syndr. 2020;14(5):797-799.

8. Fenston J. Telemedicine: Adventures in time and space [Internet]. [cited 2020 Aug 8].

9. Institute of Medicine (US) Committee on Evaluating Clinical Applications of Telemedicine. Telemedicine: A Guide to Assessing Telecommunications in Health Care [Internet]. Field MJ, editor. Washington (DC): National Academies Press (US); 1996 [cited 2020 Aug 8]. (The National Academies Collection: Reports funded by National Institutes of Health).

10. How Telemedicine Is Changing Disaster Relief [Internet]. [cited 2020 Aug 8].

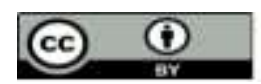

This work is licensed under Creative Commons Attribution 4.0 License

To Submit Your Article Click Here: Submit Article

DOI:10.31579/2641-0419/107
Ready to submit your research? Choose Auctores and benefit from:

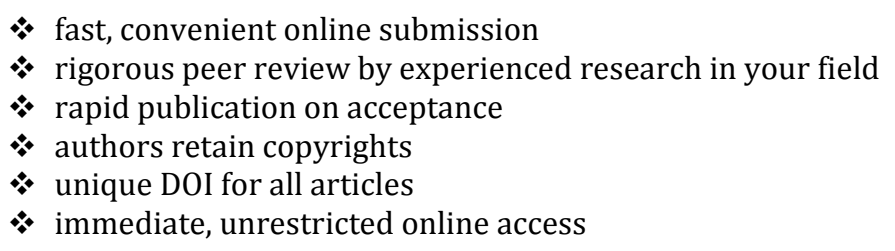

At Auctores, research is always in progress.

Learn more www.auctoresonline.org/journals/clinical-cardiology-andcardiovascular-interventions 